

\title{
Quantitative detection of codeine in human plasma using surface-enhanced Raman scattering via adaptation of the isotopic labelling principle $†$
}

\author{
Abdu Subaihi, ${ }^{a}$ Howbeer Muhamadali, ${ }^{a}$ Shaun T. Mutter, ${ }^{\text {a,c }}$ Ewan \\ Blanch, ${ }^{b}$ David I. Ellis ${ }^{\mathrm{a}}$ and Royston Goodacre ${ }^{\star a}$
}

In this study surface enhanced Raman scattering (SERS) combined with the isotopic labelling (IL) principle has been used for the quantification of codeine spiked into both water and human plasma. Multivariate statistical approaches were employed for the analysis of these SERS spectral data, particularly partial least squares regression (PLSR) which was used to generate models using the full SERS spectral data for quantification of codeine with, and without, an internal isotopic labelled standard. The PLSR models provided accurate codeine quantification in water and human plasma with high prediction accuracy $\left(Q^{2}\right)$. In addition, the employment of codeine-d6 as the internal standard further improved the accuracy of the model, by increasing the $Q^{2}$ from 0.89 to 0.94 and decreasing the low root-mean-square error of predictions (RMSEP) from 11.36 to 8.44 . Using the peak area at $1281 \mathrm{~cm}^{-1}$ assigned to $\mathrm{C}-\mathrm{N}$ stretching, $\mathrm{C}-\mathrm{H}$ wagging and ring breathing, the limit of detection was calculated in both water and human plasma to be 0.7 $\mu \mathrm{M}\left(209.55 \mathrm{ng} \mathrm{mL}^{-1}\right)$ and $1.39 \mu \mathrm{M}\left(416.12 \mathrm{ng} \mathrm{mL}^{-1}\right)$, respectively. Due to a lack of definitive codeine vibrational assignments, density functional theory (DFT) calculations have also been used to assign the spectral bands with their corresponding vibrational modes, which were in excellent agreement with our experimental Raman and SERS findings. Thus, we have successfully demonstrated the application of SERS with isotope labelling for the absolute quantification of codeine in human plasma for the first time with a high degree of accuracy and reproducibility. The use of the IL principle which employs an isotopolog (that is to say, a molecule which is only different by the substitution of atoms by isotopes) improves quantifi-cation and reproducibility because the competition of the codeine and codeine-d6 for the metal surface used for SERS is equal and this will off set any difference in the number of particles under analysis or any

fluctuations in laser fluence. It is our belief that this may open up new exciting opportunities for testing SERS in real-world samples and applications which would be an area of potential future studies.

\section{Introduction}

Codeine (3-methylmorphine) is an organic compound belong-ing to a group of medicines commonly known as opioids. In many countries codeine is regulated under narcotic laws. Within the UK for example, codeine in its various forms is regulated by the Misuse of Drugs Act (1971) as a class B con-trolled substance, with only lower-strength combinations con-taining a maximum of $12.8 \mathrm{mg}$ of codeine per dosage available

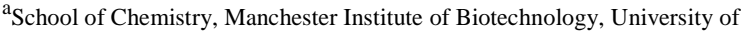
Manchester, 131 Princess Street, Manchester, M1 7DN, UK.

E-mail: roy.goodacre@manchester.ac.uk

${ }^{b}$ School of Science, RMIT, GPO Box 2476, Melbourne, Victoria 3001, Australia

${ }^{\mathrm{c} D e p a r t m e n t}$ of Chemistry, Cardiff University, Cardiff, Wales
}

over the counter without a prescription. ${ }^{1}$ Codeine is a potent analgesic drug which is commonly used as a therapeutic agent for the treatment of low, moderate and chronic pain. However, longterm use of codeine may also lead to addiction and over-doses can even cause severe liver damage, disorders and res-piratory depression. ${ }^{2,3}$

Various analytical approaches have been reported for the detection of codeine in biological fluids such as gas chromatography tandem mass spectrometry (GC-MS), ${ }^{4-6}$ high perform-ance liquid chromatography (HPLC) coupled to either ultra-violet detection $^{7,8}$ or tandem mass spectrometric detection (HPLC-MS), ${ }^{9}$ thin-layer chromatography (TLC) ${ }^{10}$ and capillary electrophoresis (CE). ${ }^{11}$ However, these well-established methods have some drawbacks, such as the requirement for lengthy sample processing steps (e.g. liquid-liquid extrac-tion or solid-phase extraction) to improve sensitivity and selectivity, requiring skilled laboratory personnel and complex 
instrumentation. Therefore, it would be advantageous for many applications to develop simple, rapid, accurate, sensitive, relatively inexpensive, portable and on-site detection approaches that are capable of monitoring drugs in various biological fluids. ${ }^{12}$

Raman spectroscopy is a physicochemical vibrational spectroscopic technique that is based on the inelastic scatter-ing of light when it interacts with molecules, providing highly specific molecular fingerprints of the sample under

analysis, which can be used for the accurate characterisation of analytes. ${ }^{13,14}$ However, due to the inherently weak Raman

signal, typically only 1 in $10^{6}-10^{8}$ photons are inelastically scattered; therefore, surface-enhanced Raman scattering (SERS) has been explored to overcome this issue by signifi-cantly improving the Raman signal through adsorption of an analyte of interest onto a roughened metal surface, which enhance the Raman effect by several orders of magnitude

yielding an ultra-sensitive and chemically selective analytical tool.15,16

SERS has recently been applied to the analysis of various complex biological samples including: bacterial cells, ${ }^{17,18}$ wholeblood and its components ( plasma, sera and red blood cells) ${ }^{19}$ and for the study of various diseases using blood serum and plasma. ${ }^{20-24}$ Several studies have reported the application of SERS for the successful detection, identification and quantification of illicit drugs. $^{25,26}$ Alharbi and colleagues also used this approach for the quantitative detection of the opioid tramadol in artificial urine. ${ }^{27}$ Dong et al. employed (dynamic) D-SERS and support vector machines for the quanti-tative detection of methamphetamine in human urine. ${ }^{28}$ Andreou et al. also reported the detection of methamphet-amine in saliva samples using a microfluidic device which controlled the interaction between the drug, Ag nanoparticles, and the aggregation agent. ${ }^{29}$ Finally, in a more recent study, we have also demonstrated SERS as a powerful technique for the quantitative detection of the beta-blocker propranolol in human biofluids (serum, plasma, urine) when combined with chemometric approaches. ${ }^{30}$

However, the application of SERS for the detection and quantification of drugs in complex biological media can face several challenges, most notably the reproducibility, accuracy and repeatability of the SERS signal which could be due to ran-domly distributed hot spots which occur during sample ana-lysis. ${ }^{31,32}$ Therefore, in this study the IL principle combined with a portable Raman spectrometer has been employed to improve the quantitative detection of codeine in human plasma. This approach is based on the addition of a known constant quantity of isotopically labelled codeine (codeine- $\mathrm{d}_{6}$ ) as an internal standard. We show that this could be employed in a portable assay for the detection of such drugs at very low concentration in human biofluids. Our results clearly demon-strate the potential application of this strategy, combining IL with SERS, for development of quantitative detection of codeine in human plasma, providing a highly specific and accurate prediction of codeine concentrations with minimal error.

\section{Materials and methods}

\section{Materials}

Silver nitrate $(99.9 \%)$, trisodium citrate, sodium chloride, codeine, codeine-d6 and human plasma were all purchased from Sigma Aldrich (Dorset, United Kingdom). All plasma samples were sourced from the same analytical batch to reduce any batch-to-batch variation. Amicon Ultra $0.5 \mathrm{~mL}$ cen-trifugal filters $(3 \mathrm{kDa})$ were purchased from Merck Millipore Ltd (Germany). All chemicals used were of analytical grade.

Synthesis of silver nanoparticles

The Lee and Meisel citrate reduction method ${ }^{33}$ was used to prepare the silver nanoparticles (AgNPs). Briefly, $90 \mathrm{mg}$ of $\mathrm{AgNO}_{3}$ was dissolved in $500 \mathrm{~mL}$ of deionised water; the solu-tion was heated to boiling point, $1 \%$ aqueous solution of tri-sodium citrate $(10 \mathrm{~mL})$ was added to a stirring silver nitrate solution drop by drop. The mixed solution was left at boiling point for a further 15 min until a greenmilky silver colloid was observed, which proved to be stable and useable for several weeks following preparation. The colloid was assessed using UV-visible spectroscopy and scanning electron microscopy (SEM) and was very similar to that used in previously pub-lished studies. $^{25,26,34,35}$

\section{Instrumentation}

Initial experiments were carried out to generate conventional Raman spectra of powdered codeine and codeine- $\mathrm{d}_{6}$, which were placed on a calcium fluoride $\left(\mathrm{CaF}_{2}\right)$ disc and Raman spectra were recorded using $633 \mathrm{~nm}$ excitation (see ESI† for more details of the conventional Raman measurements).

SERS spectra were recorded using a DeltaNu Advantage portable Raman spectrometer (DeltaNu, Laramie, WY, USA), equipped with a $633 \mathrm{~nm}$ HeNe laser emitting $\sim 3 \mathrm{~mW}$ on to the sample. Daily calibration of the instrument was performed using a polystyrene internal standard as supplied by the instru-ment manufacturer. All samples were analysed within the 400 to $3400 \mathrm{~cm}^{-1}$ spectral range, with a spectral resolution of $8 \mathrm{~cm}^{-1}$ and the laser exposure time was set to $20 \mathrm{~s}$ for all samples.

\section{Sample preparation for SERS measurements}

Stock solutions (10 mL each) of human plasma and distilled water were spiked individually each with $1.5 \mathrm{mg}$ of codeine and codeined6. A serial dilution of unlabelled codeine with plasma (no water added) and water was then prepared at a range of concentrations for quantitative analysis, while the concentration of the stable isotope standard (codeine- $\mathrm{d}_{6}$ ) was kept fixed. Mixtures of either water or plasma spiked with unlabelled and labelled codeine were prepared at diff erent codeine concentrations ranging from $0 \mu \mathrm{M}$ to $140 \mu \mathrm{M}$. Amicon ultra-centrifugal filters were used to remove protein residues from plasma samples, $0.5 \mathrm{~mL}$ aliquots were transferred onto the filter tube and centrifuged at $14000 \mathrm{~g}$ for $30 \mathrm{~min}$ following the manufacturer's recommended protocol. All plasma samples were then stored at $-80{ }^{\circ} \mathrm{C}$ until required for analysis. 
To minimise any SERS signal variability as a result of differing volumes of components, the colloid/analyte volume were kept at 200 $\mu \mathrm{L}$ each and $\mathrm{NaCl}$ as an aggregation agent at $50 \mu \mathrm{L}$, resulting in a total $450 \mu \mathrm{L}$ of solution for each sample. The resulting mixture was vortexed for $2 \mathrm{~s}$ and placed into the sample cell attachment, which was immediately interrogated by laser irradiation for $20 \mathrm{~s}$. A total of five biological replicates and two analytical replicates were prepared for each biological matrix and for each concentration.

\section{Chemometrics SERS data processing}

MATLAB software R2013a (The Math Works Inc, Natwick, USA) was used to process all SERS spectral data. All SERS data were baseline corrected using asymmetric least squares (AsLS) ${ }^{36}$ and subjected to the supervised method of partial least squares regression (PLSR). ${ }^{37,38}$ PLSR is a multivariate quantitative regression technique generally used for predictive linear mod-elling, as reported previously by our research group. ${ }^{39,40}$ PLSR was also employed to generate separate models for predicting the concentration of codeine spiked into either water or plasma. The PLSR models were constructed using the entire SERS spectral data. Four separate PLSR models were generated from: (i) codeine spiked in water, (ii) a mixture of codeine/ codeine-d6 spiked in water, (iii) codeine spiked in plasma, (iv) a mixture of codeine/codeine-d6 spiked in plasma. For each PLSR model, $50 \%$ of the data were used as the training set to calibrate the multivariate model. Once the models were cali-brated, the remaining data (test set) were used for the predic-tion of codeine concentrations and also were used for vali-dation purposes.

\section{Results and discussion}

Preliminary investigations were conducted using Raman spectroscopy with $633 \mathrm{~nm}$ excitation to analyse powdered samples (2-3 $\mu \mathrm{g}$ ) of codeine and codeine- $\mathrm{d}_{6}$. The chemical structures of these compounds are presented in Fig. 1, and the resulting Raman spectra are provided in (Fig. S1†) which illustrates the spectral shifts as a result of changes in the reduced mass of vibrations that involved deuterium (D, $\left.{ }^{2} \mathrm{H}\right)$ instead of hydro-gen. Due to limited information on Raman spectral band assignments of codeine and codeine- $\mathrm{d}_{6}$, quantum chemical

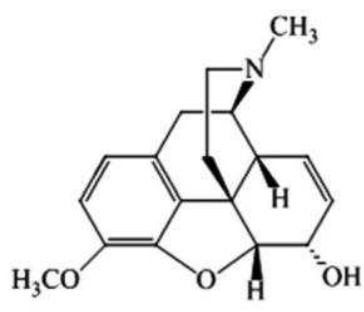

Codeine

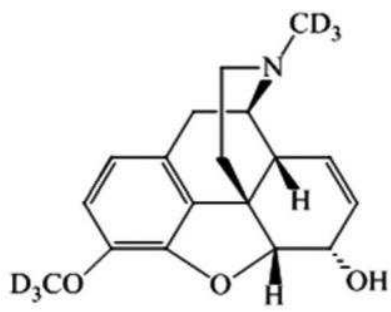

Codeine- $d 6$ calculations based on density functional theory (DFT) have been used to confirm these peak shifts and their corres-ponding vibrational band assignments; we refer the reader to the ESI† for more details on the DFT calculations. The calcu-lated spectra were generated based on a gas phase environ-ment (Fig. S2†), but even with this assumption the details of the modelled spectrum are clearly in agreement with our experimental findings. Table S1† provides tentative assign-ments of the main bands affected by the labelling process, which can be assigned to the $\mathrm{CH}$ stretching (2800 to 3200 $\mathrm{cm}^{-1}$ ) region of unlabelled codeine, that are shifted to the $\mathrm{CD}$ stretching (2070 to $2260 \mathrm{~cm}^{-1}$ ) region for the codeine sample labelled with deuterium. As the concentrations of codeine that we wish to detect from human plasma are too low to be observed using conventional Raman, due to the inherent weakness of the Raman effect mentioned above, SERS was therefore employed to improve the sensitivity and lower the detection limit of codeine in the biofluids under investigation.

Initial optimizations of the SERS parameters for codeine detection were carried out by examining various factors, such as: the forms of metal nanoparticles, the aggregation agent, as well as the aggregation time used. Subsequently, the optimal conditions for SERS enhancement were achieved using a citrate-reduced silver colloid with sodium chloride as the aggregation agent which is similar to our previously published protocol. ${ }^{30}$ A previous study reported the SERS optimum con-ditions of codeine dissolved in water, ${ }^{41}$ where silver nano-particles with $\mathrm{NaCl}$ gave the highest enhancement at $633 \mathrm{~nm}$ and which further supported the optimization conditions used in our present study.

Prior to analysing codeine in complex human plasma which contains many proteins and small molecules that may interfere with SERS, codeine was initially investigated in water, to reduce the complexity of the resulting spectra and also to facilitate accurate identification of the SERS peak shifts in samples containing labelled codeine. SERS spectra of codeine and codeine-d6 spiked into water are presented in Fig. 2a. The main bands affected by the presence of the heavy isotope were found to be at 2953, 3028 and $3059 \mathrm{~cm}^{-1}$, corresponding to the symmetric $\mathrm{C}-\mathrm{H}$ stretching of $\mathrm{N}-\mathrm{CH}_{3}$, symmetric $\mathrm{C}-\mathrm{H}$ stretching of $\mathrm{O}-\mathrm{CH}_{3}$ and asymmetric $\mathrm{C}-\mathrm{H}$ stretching of $\mathrm{O}-\mathrm{CH}_{3}$, respectively. These were shifted to 2075,2128 and $2275 \mathrm{~cm}^{-1}$, with these vibrations being assigned to $\mathrm{N}-\mathrm{CD}_{3}, \mathrm{O}-$ $\mathrm{CD}_{3}$ and $\mathrm{O}-\mathrm{CD}_{3}$ in codeine-d6. A list of the main bands detected, and their corresponding assignments, are summar-ized in Table S1.† As shown previously, SERS of analytes directly in plasma was not possible. ${ }^{30}$ This is largely due to the high abundance of proteins in plasma (e.g., albumin) which may interfere with the aggregation of the SERS nanoparticles. Therefore, ultra-centrifugal filters were used to minimise protein content in these samples, a strategy we have very recently used successfully and further details of this can be found in the literature. ${ }^{30}$ The SERS spectra of codeine and codeine-d6 in plasma (see Fig. 2b) displayed similar peak shifts described above resulting from the presence of the heavy isotope.

Fig. 1 The chemical structures of codeine and codeine-d6 showing where $\mathrm{CH}_{3}$ were replaced with $\mathrm{CD}_{3}$. 

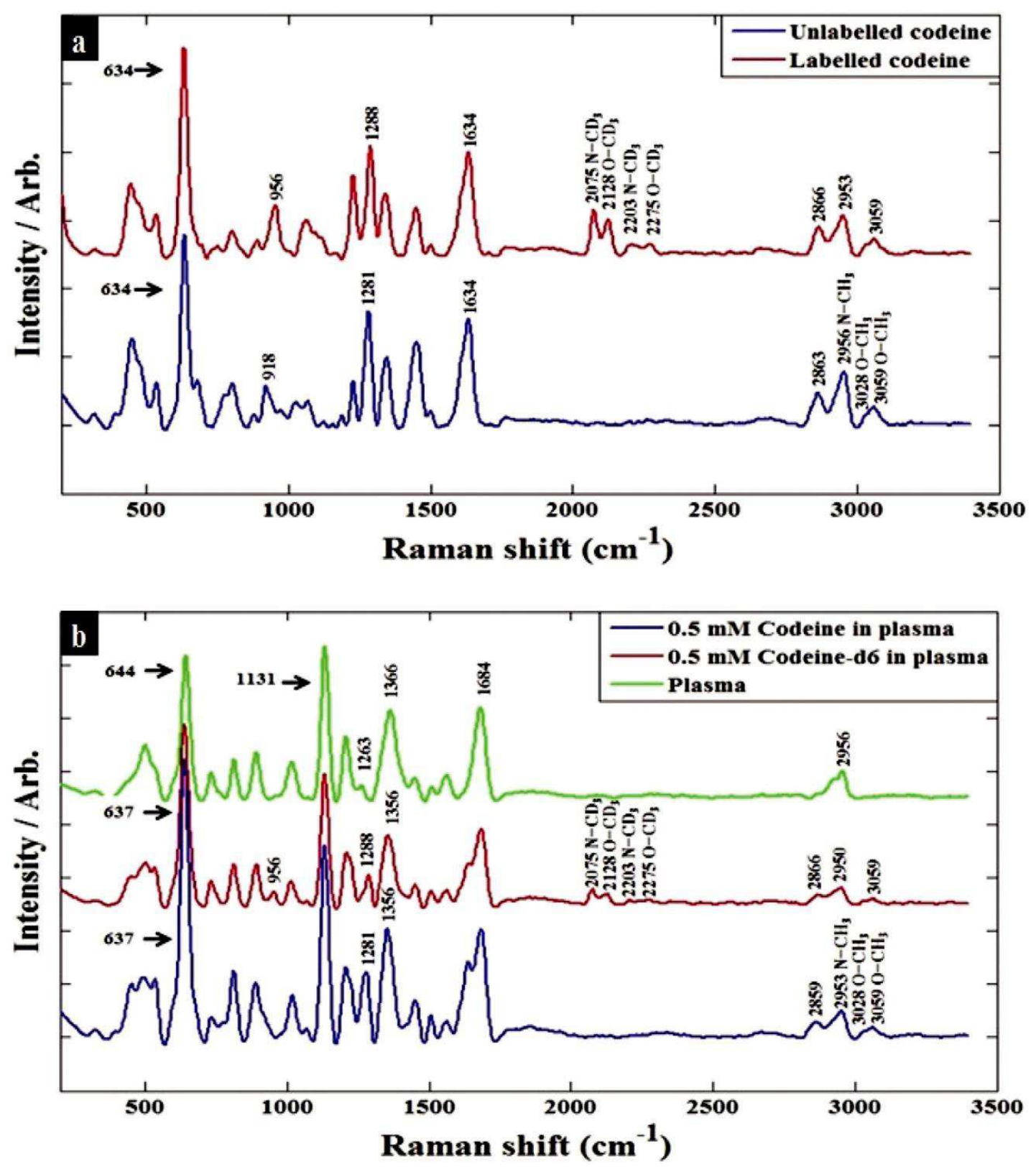

Fig. 2 Baseline-corrected SERS spectra of $100 \mu \mathrm{M}$ codeine spiked into (a) water (b) human plasma. The blue spectra refer to codeine, red spectra belong to codeine-d6, and the green spectrum is collected from pure plasma. Annotations with arrow are bands that are assigned to specific vibrational modes (see Tables S1 and S2†).

There were many features observed in the SERS spectra of pure plasma, the most significant peaks that could be assigned to plasma were at 644 and $1131 \mathrm{~cm}^{-1}$, which are attributed to tyrosine and Dmannose, respectively; ${ }^{20,30}$ Table $\mathrm{S} 2 \dagger$ summar-izes the main band assignments for pure plasma. In order to examine the limit of detection (LOD) of codeine in both water and plasma, the peak area at $1281 \mathrm{~cm}^{-1}$ (assigned to $\mathrm{C}-\mathrm{N}$ stretch-ing, $\mathrm{C}-\mathrm{H}$ wagging and ring breathing) was used to calculate the LOD, by plotting the area under this peak with varying concen-trations of codeine (Fig. S3a and b†). In these figures there is a slight sigmoidal shape to the curve for codeine, which is more accentuated in plasma compared to water. This is likely to be due to increased competition of the codeine for the surface of the colloid which, when it is present in the plasma, is competing with other components (viz. organic acids, salts, metabolites etc.) found naturally within human blood plasma. Based on 3 times the standard deviation of the response and the slope, ${ }^{42}$ the LOD in water and plasma were found to be $0.7 \mu \mathrm{M}\left(209.55 \mathrm{ng} \mathrm{mL} \mathrm{m}^{-1}\right)$ and $1.39 \mu \mathrm{M}$ (416.12 $\mathrm{ng} \mathrm{mL}^{-1}$ ), respectively. The higher LOD for codeine in plasma is for reasons just mentioned perhaps not surprising and is likely to be due to the presence of various mole-cular species in the plasma competing for binding with the metal surface, and restricting the interaction of the codeine molecules with the metal nanoparticles. ${ }^{43,44}$ 

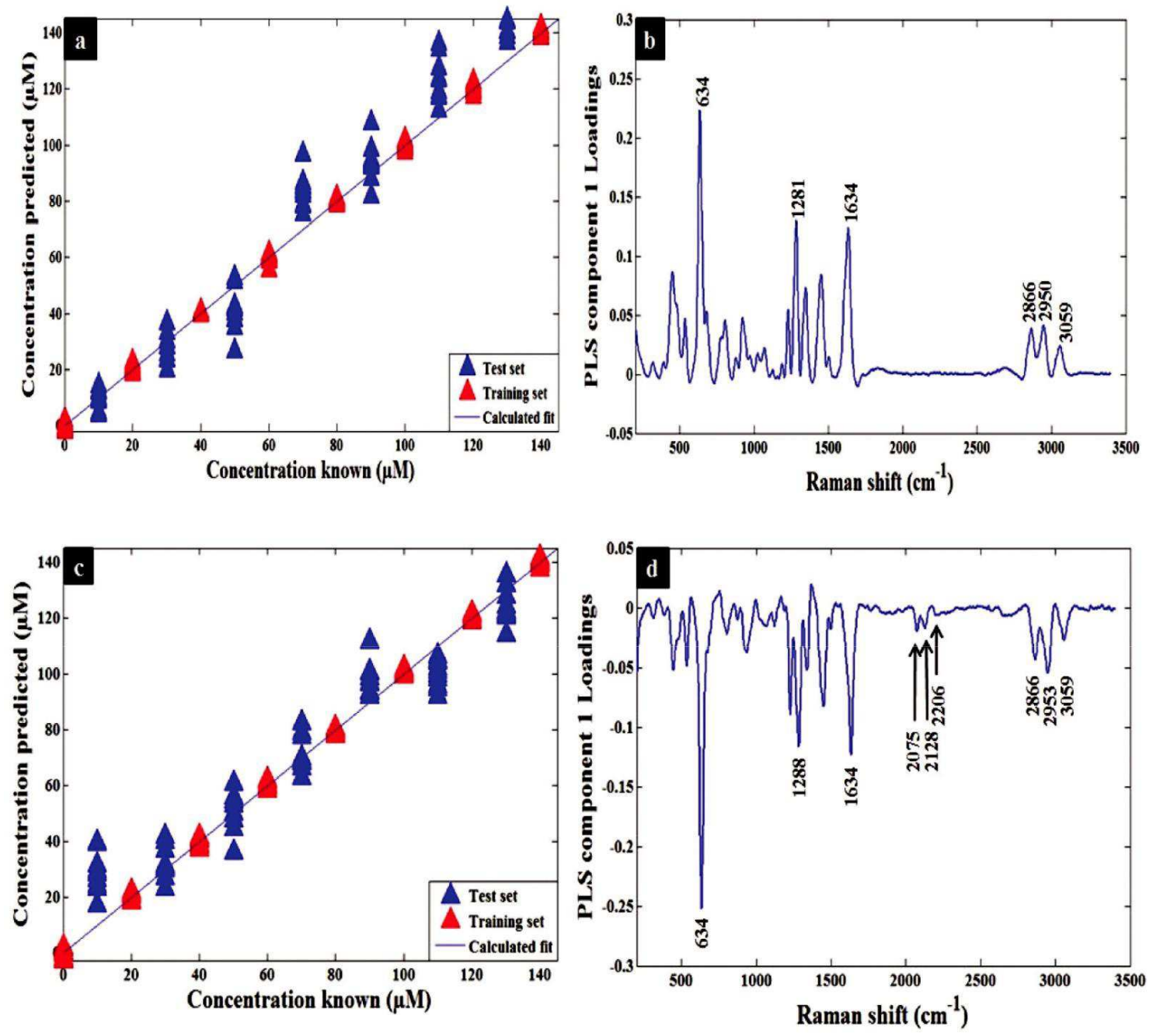

Fig. 3 Representative PLS based predictions of concentration versus known concentrations of (a) codeine and (c) mixtures of codeine + codeine-d6 spiked in water. Note that the concentration of codeine-d6 remained constant. The corresponding PLS loading plots are from (b) codeine alone, and (d) after employing the isotopic labelling with codeine-d6.

Quantification of codeine in water and plasma samples were carried out by generating PLSR models using the whole SERS spectral data as described in the chemometrics section. The quantitative models were generated and calibrated using the SERS data of codeine alone and mixtures of codeine/ codeine-d 6 spiked into water (Fig. 3a and c). A similar approach was also employed for the analysis of codeine and mixtures of codeine/codeine-d 6 spiked into human plasma (Fig. 4a and c). The generated models demonstrated a slight improvement of prediction accuracy $\left(Q^{2}\right)$ from 0.74 to 0.79 and from 0.89 to 0.94 in water and human plasma, respectively, when using the isotopic labelling approach. In addition, using this approach the root-mean-square error of prediction (RMSEP) also improved (i.e. decreased): from 11.41 to 7.75 and 18.74 to 12.71 in water and human plasma, respectively. It is noted that the deviations of some samples are much larger than others in Fig. 4a and $\mathrm{c}$ and they are more likely due to the experimental factors. Table 1 contains a summary of the statistics for the PLSR models and also details the same stati- stics for the training set (viz. $\mathrm{R}^{2}$ and RMSECV). Additionally, the PLS component loadings are plotted to confirm the above findings by revealing the contributed bands of labelled codeine as peaks, at 2075, 2128 and $2275 \mathrm{~cm}^{-1}$, as these were the main significant variables influenced by codeine-d6 in either water (Fig. $3 \mathrm{~b}$ and d) or plasma (Fig. $4 \mathrm{~b}$ and d).

\section{Conclusion}

Here, we have developed a simple and robust SERS-based method for the quantitative detection of the opioid codeine in water and human plasma. In addition, the application of the IL principle also improved the accuracy and precision of this approach. We have shown that the use of the IL principle by the addition of an isotopolog (i.e., a molecule which is only different by the substitution of atoms by isotopes) improves quantification and reproducibility because the competition of the codeine and codeine$\mathrm{d}_{6}$ for the metal surface used for SERS 

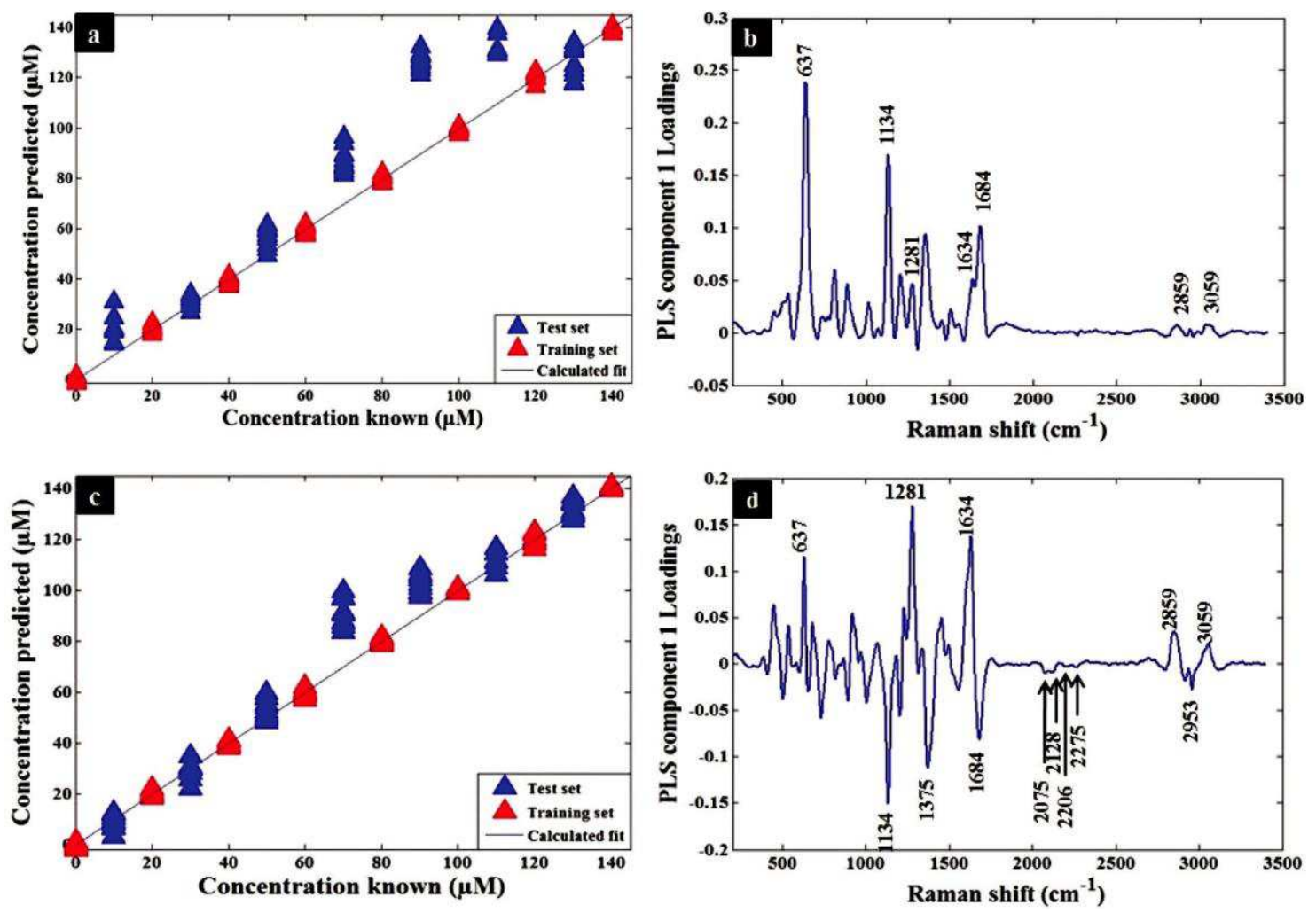

Fig. 4 Representative PLS based predictions of concentration versus known concentrations of (a) codeine and (c) mixtures of codeine + codeine-d6 spiked in plasma. Note that the concentration of codeine-d6 remained constant. The corresponding PLS loading plots are from (b) codeine alone, and (d) after employing the isotopic labelling with codeine-d6.

Table 1 Comparison of PLSR models showing their reproducibility and accuracy of predicting codeine concentrations spiked to water and plasma

\begin{tabular}{|c|c|c|c|c|}
\hline \multirow[b]{2}{*}{ Metric } & \multicolumn{2}{|l|}{ Water } & \multicolumn{2}{|l|}{ Plasma } \\
\hline & Codeine & $\begin{array}{l}\text { Codeine }+ \\
\text { codeine- } \mathrm{d}_{6}\end{array}$ & Codeine & $\begin{array}{l}\text { Codeine }+ \\
\text { codeine- } \mathrm{d}_{6}\end{array}$ \\
\hline Factors & 9 & 9 & 9 & 9 \\
\hline $\mathrm{R}^{2}$ & 0.9993 & 0.9993 & 0.9993 & 0.9994 \\
\hline $\mathrm{Q}^{2}$ & 0.7418 & 0.7898 & 0.8895 & 0.9389 \\
\hline RMSEP & 11.4107 & 7.7543 & 18.7416 & 12.7135 \\
\hline RMSECV & 11.4655 & 8.6613 & 11.3560 & 8.4436 \\
\hline
\end{tabular}

Factors specify the number of latent variables used in PLSR; RMSEP, rootmean-squared error of prediction (from the test set); RMSECV root mean squared error of cross validation (from the training set); $\mathrm{R}^{2}$ and $\mathrm{Q}^{2}$ show linearity for the training and test sets predictions respectively.

is equal and this will compensate for any diff erence in the number of nanoparticles under analysis or any fluctuations in laser fluence which are known to affect quantification. The main bands aff ected by the presence of the heavy isotope were found to be at 2953, 3028 and $3059 \mathrm{~cm}^{-1}$ corresponding to the symmetric $\mathrm{C}-\mathrm{H}$ stretching of $\mathrm{N}-\mathrm{CH}_{3}$, symmetric $\mathrm{C}-\mathrm{H}$ stretching of $\mathrm{O}-\mathrm{CH}_{3}$, and asymmetric $\mathrm{C}-\mathrm{H}$ stretching of $\mathrm{O}-\mathrm{CH}_{3}$, respect-ively. These were shifted to 2075 , 2128 and $2275 \mathrm{~cm}^{-1}$ assigned to $\mathrm{N}-\mathrm{CD}_{3}, \mathrm{O}-\mathrm{CD}_{3}$ and $\mathrm{O}-\mathrm{CD}_{3}$ in the codeine-d6 isotopolog. DFT calculations were employed in order to support the vibrational assignments of codeine and codeine- $\mathrm{d}_{6}$ and these were in agree-ment with our experimental findings. The LOD of codeine in both water and plasma were $0.7 \mu \mathrm{M}\left(209.55 \mathrm{ng} \mathrm{mL}^{-1}\right)$ and $1.39 \mu \mathrm{M}(416.12 \mathrm{ng}$ $\mathrm{mL}^{-1}$ ), respectively. Future work is required to improve the LOD of the codeine SERS signal to reach the detection level that is expected for clinical samples (9.7 to $210 \mathrm{ng} \mathrm{mL}^{-1}$ ), ${ }^{45}$ where we could use superhydrophobic wires with hydrophilic tips which would allow us to improve the LOD of codeine ${ }^{46}$ or concentrate the sample prior to analyses by evaporation. Despite the fact that the detection limit is not yet clinically useful these findings clearly demonstrate the potential application of SERS when combined with internal isotope stan-dards as a portable technique that could be readily and remotely deployed for on-site testing in clinical settings.

\section{Acknowledgements}

A. S. thanks The Saudi Ministry of High Education and Umm alQura University for funding. R. G. is indebted to UK. BBSRC (Grant BB/L014823/1) for funding for Raman spectroscopy. Human plasma from pooled human blood was obtained from Sigma Aldrich (Dorset, United Kingdom) and therefore, full ethics following the 1964 Declaration of Helsinki was not required for this study. 


\section{References}

1 S. Bradshaw, Br. Med. J., 1971, 4, 363.

2 M. Häkkinen, T. Launiainen, E. Vuori and I. Ojanperä, Forensic Sci. Int., 2012, 222, 327-331.

3 B. A. Sproule, U. E. Busto, G. Somer, M. K. Romach and E. M. Sellers, J. Clin. Psychopharmacol., 1999, 19, 367-372.

4 M. Krogh, A. S. Christophersen and K. E. Rasmussen, J. Chromatogr. B: Biomed. Sci. Appl., 1993, 621, 41-48.

5 K. A. Rees, P. A. McLaughlin and M. D. Osselton, J. Anal. Toxicol., 2012, 36, 1-11.

6 U. Hofmann, S. Seefried, E. Schweizer, T. Ebner, G. Mikus and M. Eichelbaum, J. Chromatogr. B: Biomed. Sci. Appl., 1999, 727, 81-88.

7 H. He, S. D. Shay, Y. Caraco, M. Wood and A. J. Wood, J. Chromatogr. B: Biomed. Sci. Appl., 1998, 708, 185-193.

8 M. Shamsipur and N. Fattahi, J. Chromatogr. B: Anal. Technol. Biomed. Life Sci., 2011, 879, 2978-2983.

9 Z. Hu, Q. Zou, J. Tian, L. Sun and Z. Zhang, J. Chromatogr. B: Anal. Technol. Biomed. Life Sci., 2011, 879, 3937-3942.

10 D.-S. Popa, R. Oprean, E. Curea and N. Preda, J. Pharm. Biomed. Anal., 1998, 18, 645-650.

11 M.-E. Capella-Peiró, D. Bose, M. F. Rubert and J. EsteveRomero, J. Chromatogr. B: Anal. Technol. Biomed. Life Sci., 2006, 839, 95-101.

12 A. Bonifacio, S. Cervo and V. Sergo, Anal. Bioanal. Chem., 2015, 407, 8265-8277.

13 D. I. Ellis, D. P. Cowcher, L. Ashton, S. O'Hagan and R. Goodacre, Analyst, 2013, 138, 3871-3884.

14 D. I. Ellis and R. Goodacre, Analyst, 2006, 131, 875-885.

15 A. Campion and P. Kambhampati, Chem. Soc. Rev., 1998, 27, 241-250.

16 M. Fleischmann, P. J. Hendra and A. McQuillan, Chem. Phys. Lett., 1974, 26, 163-166.

17 H. Muhamadali, A. Subaihi, M. Mohammadtaheri, Y. Xu, D. I. Ellis, R. Ramanathan, V. Bansal and R. Goodacre, Analyst, 2016, 141, 5127-5136.

18 R. M. Jarvis and R. Goodacre, Anal. Chem., 2004, 76, 40-47.

19 W. Premasiri, J. Lee and L. Ziegler, J. Phys. Chem. B, 2012, 116, 9376-9386.

20 S. Feng, R. Chen, J. Lin, J. Pan, G. Chen, Y. Li, M. Cheng, Z. Huang, J. Chen and H. Zeng, Biosens. Bioelectron., 2010, 25, 2414-2419.

21 S. Feng, R. Chen, J. Lin, J. Pan, Y. Wu, Y. Li, J. Chen and H. Zeng, Biosens. Bioelectron., 2011, 26, 3167-3174.

22 D. Lin, S. Feng, J. Pan, Y. Chen, J. Lin, G. Chen, S. Xie, H. Zeng and R. Chen, Opt. Express, 2011, 19, 13565-13577.
23 J. Lin, R. Chen, S. Feng, J. Pan, B. Li, G. Chen, S. Lin, C. Li, L. Q. Sun and Z. Huang, J. Raman Spectrosc., 2012, 43, 497502.

24 S. Feng, D. Lin, J. Lin, B. Li, Z. Huang, G. Chen, W. Zhang, L. Wang, J. Pan and R. Chen, Analyst, 2013, 138, 3967- 3974.

25 S. Mabbott, E. Correa, D. P. Cowcher, J. W. Allwood and R. Goodacre, Anal. Chem., 2012, 85, 923-931.

26 S. Mabbott, O. Alharbi, K. Groves and R. Goodacre, Analyst, 2015, 140, 4399-4406.

27 O. Alharbi, Y. Xu and R. Goodacre, Analyst, 2015, 140, 59655970.

28 R. Dong, S. Weng, L. Yang and J. Liu, Anal. Chem., 2015, 87, 2937-2944.

29 C. Andreou, M. R. Hoonejani, M. R. Barmi, M. Moskovits and C. D. Meinhart, ACS Nano, 2013, 7, 7157-7164.

30 A. Subaihi, L. Almanqur, H. Muhamadali, N. Almasoud, D. I. Ellis, D. K. Trivedi, K. A. Hollywood, Y. Xu and R. Goodacre, Anal. Chem., 2016, 88, 10884-10892.

31 Y. Fang, N.-H. Seong and D. D. Dlott, Science, 2008, 321, 388392.

32 R. Aroca, R. Alvarez-Puebla, N. Pieczonka, S. Sanchez-Cortez and J. Garcia-Ramos, Adv. Colloid Interface Sci., 2005, 116, 4561.

33 P. Lee and D. Meisel, J. Phys. Chem., 1982, 86, 3391-3395.

34 D. P. Cowcher, Y. Xu and R. Goodacre, Anal. Chem., 2013, 85, 3297-3302.

35 O. Alharbi, Y. Xu and R. Goodacre, Analyst, 2014, 139, 48204827.

36 P. H. Eilers, Anal. Chem., 2004, 76, 404-411.

37 P. Geladi and B. R. Kowalski, Anal. Chim. Acta, 1986, 185, 117.

38 H. Martens and T. Naes, Multivariate calibration, John Wiley \& Sons, 1992.

39 H. Muhamadali, M. Chisanga, A. Subaihi and R. Goodacre, Anal. Chem., 2015, 87, 4578-4586.

40 N. Nicolaou, Y. Xu and R. Goodacre, Anal. Chem., 2011, 83, 5681-5687.

41 V. Rana, M. V. Cañamares, T. Kubic, M. Leona and J. R.

Lombardi, J. Forensic Sci., 2011, 56, 200-207.

42 I. H. T. Guideline, Q2 (R1), 2005, 1.

43 J. Judis, J. Pharm. Sci., 1977, 66, 802-806.

44 J. Judis, J. Pharm. Sci., 1980, 69, 71-73.

45 J. W. Findlay, E. C. Jones, R. F. Butz and R. M. Welch, Clin. Pharmacol. Ther., 1978, 24, 60-68.

46 M. Cheung, W. W. Lee, D. P. Cowcher, R. Goodacre and S. E. Bell, Chem. Commun., 2016, 52, 9925-9928. 